\title{
FACTORES RELACIONADOS CON LA PRESENCIA DE PERROS EN LAS CALLES DE LA CIUDAD DE SANTIAGO, CHILE
}

\author{
FACTORS RELATED TO THE PRESENCE OF DOGS \\ IN THE STREETS OF SANTIAGO CITY, CHILE
}

\author{
LUIS IBARRA ${ }^{1} ;$ FABIÁN ESPÍNOLA $^{1} ;$ MARIO ECHEVERRÍA ${ }^{1}$
}

\begin{abstract}
In roles to know which are the factors related to the dogs in the streets and as a contribution to designing of sanitary measures for canine population surveillance and controlling, a research was carried out in 34 districts from the Metropolitan Region that conform Santiago city. The sampling unit was the street block and the specific information was obtained through direct observation of 864 city blocks from Santiago, by giving a minimum of 25 city blocks by district, randomly chosen. The street canine population characteristics, the urban structure and the habitat were registered to investigate the relationship among them.

The results show that the amount and types of dogs observed in the streets of Santiago are related to the existence of "shelter like" places and water and food sources in the urban structure of Santiago. The presence of dogs is also related to the garbage location in the street design, because the amount of dogs is significantly smaller if there are storing facilities or baskets in height that prevent the animal access to the garbage.
\end{abstract}

KEY WORDS: Stray dogs, free roaming dogs, environment.

Palabras Clave: Perros vagabundos, perros callejeros, ambiente.

\section{INTRODUCCIÓN}

Debido a lo atractiva y beneficiosa que puede resultar la relación del ser humano con el perro, es que una gran cantidad de personas optan por adoptar este animal como mascota. Se debe reconocer, a la vez, que la convivencia con los animales domésticos genera en la población humana una serie de problemas. Específicamente se pueden mencionar los problemas originados por los perros vagabundos y callejeros en la vía pública, tales como mordeduras provocadas a las personas, la ruptura y vaciamiento de las bolsas de basura en busca de comida, contaminación del ambiente con deposiciones, accidentes de tránsito, etc., (Álvarez y Domínguez,

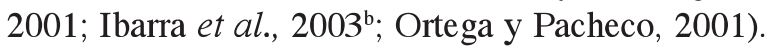
También, se debe tener en consideración las malas condiciones de vida que llevan estos animales, pues se

I Facultad de Ciencias Veterinarias y Pecuarias, Universidad de Chile. Casilla 2, Correo 15, Santiago. Email: libarra@uchile.cl encuentran expuestos a atropellos por vehículos, mala alimentación, mayor riesgo de contagio de enfermedades, falta de abrigo, reproducción descontrolada, entre otras (Morales et al., 2002).

Diversos son los factores condicionantes para la existencia en la ciudad y particularmente en las calles de un numeroso contingente de perros. Por una parte la falta de tenencia responsable de numerosos propietarios de perros que no ejercen un control adecuado de sus animales, permitiendo su permanencia en la calle o no controlando su reproducción lo que origina una población indeseada (Betzhold, 1998). Parte de esta población, muchas veces pasa a formar parte de los animales abandonados y posteriormente de la población vagabunda. Condiciones ambientales como la mala disposición de basuras, la existencia de micro basurales, proporcionan una fuente de alimento al perro vagabundo y la existencia de sitios eriazos y estructuras urbanas abiertas y abandonadas puede proporcionarles un lugar de refugio.

Si bien se dispone de información de la población de perros existente en las viviendas de la ciudad de 
Santiago (Ibarra et al., 2003 ), entre las razones por las cuales fallan las medidas destinadas a controlar la población canina, está la falta de información de las características de esta población y del ambiente que favorece su presencia, específicamente de la existente en las calles de la ciudad, lo que ha limitado y a veces impedido una buena orientación de los programas destinados a un control eficiente. Como todo quehacer científico, los estudios demográficos están destinados a descubrir las relaciones entre fenómenos que permitan explicar su conducta, y eventualmente, puedan permitir hacer proyecciones y por lo tanto se podrían tomar acciones de tipo preventivo. El objetivo que se plantea es conocer que factores se relacionan con la presencia de perros en las calles, como contribución para la formulación de medidas sanitarias de vigilancia y control de la población canina.

\section{MATERIAL Y MÉTODO}

El estudio se realizó en 34 comunas de la Región Metropolitana, Chile. De éstas, 32 pertenecen a la provincia de Santiago, a las que se suman la comuna de San Bernardo que pertenece a la provincia del Maipo y la comuna de Puente Alto perteneciente a la provincia Cordillera, conformando la ciudad de Santiago. La unidad de muestreo fue la manzana. El tamaño de muestra para Santiago, fue de $\mathrm{n}=864$ manzanas, asignando un mínimo de 25 manzanas por comuna. Se utilizó el muestreo aleatorio para la selección de las manzanas en cada comuna. La información específica de la manzana se obtuvo mediante la observación directa, durante una hora, de dos de las cuatro cuadras que la conforman, respetando una longitud de 125 metros por cuadra, para extrapolar los resultados obtenidos en esa área, al área urbana comunal y posteriormente a la ciudad. Se registraron características de la población de perros observados en la calle que se informan en otro trabajo, y características de la estructura urbana y del hábitat del lugar, básicas para este estudio y la relación entre ellas.

Con el propósito de tener una terminología clara y única se aplicaron los siguientes conceptos, en base al nivel de dependencia del perro en el cuidado humano, esto es, alimento, albergue y compañía humana, y también al nivel de restricción o supervisión impuesta al perro por las personas:

- Perro supervisado o restringido: totalmente dependiente y totalmente restringido o supervisado.
- Perro callejero: totalmente dependiente; semirestringido.

- Perro del vecindario: semi-dependiente; semirestringido o sin restricción.

- Perro vagabundo: independiente, sin restricción. (WHO-WSPA, 1990).

La comparación de promedios se realizó mediante análisis de varianza y comparaciones múltiples de Scheffé, manteniendo un nivel de significación de $5 \%$ en todas las comparaciones (Taucher, 1997). Los datos respecto al área urbana de cada comuna de la ciudad, información muy importante para el desarrollo del trabajo, fue obtenida del "Proyecto OTAS", Bases para el Ordenamiento Territorial Ambientalmente Sustentable de la Región Metropolitana de Santiago, patrocinado por el Gobierno Regional Metropolitano de Santiago.

\section{RESULTADOS Y DISCUSIÓN}

Los datos registrados en cada unidad de observación, esto es en cada manzana muestreada, se tabularon considerando el número y tipo de perros observados en el lugar, de acuerdo a los diversos factores incluidos en la pauta de observación. Se describe estadísticamente (mediante promedio y desviación típica), el número de perros observado, por tipo de perro, por tipo de hábitat, y en general por las variables de interés registradas en el estudio.

El (Cuadro 1), muestra la descripción estadística del número de perros observados por manzana, considerando el tipo de perro, clasificados según el hábitat predominante en la manzana. Al considerar el total de perros, no se detectan diferencias estadísticamente entre los promedios correspondientes a los hábitat considerados. Al realizar el análisis en los diferentes tipos de perros, no se detectan diferencias en el caso de perros vagabundos, en que el promedio es estadísticamente el mismo en todos los hábitat.

Para perros callejeros, supervisados y de vecindario si se detectan diferencias estadísticas significativas $(\mathrm{p} \leq 0,05)$ entre los hábitat. En el caso de perros callejeros, el hábitat casas $(3,8499)$ y casas-espacio abierto $(3,8667)$ presenta un promedio mayor que el resto de los hábitat. La presencia de perros supervisados en la calle se agrupan en dos tipos de hábitat, por una parte, los departamentos, mercado-comerciooficinas y las plazas presentan promedios mayores de perros supervisados en las calles que los hábitat con 
CUADRO 1

DESCRIPCIÓN DEL NÚMERO DE PERROS EN LA CALLE POR MANZANA, POR TIPO DE PERRO, SEGÚN HÁBITAT PREDOMINANTE, CIUDAD DE SANTIAGO, 2004

\begin{tabular}{|c|c|c|c|c|c|c|c|c|c|c|c|}
\hline \multirow{2}{*}{$\begin{array}{c}\text { Hábitat } \\
\text { Predominante }\end{array}$} & \multirow{2}{*}{$\begin{array}{c}N \\
862\end{array}$} & \multicolumn{2}{|c|}{ Callejero } & \multicolumn{2}{|c|}{ Supervisado } & \multicolumn{2}{|c|}{ Vagabundo } & \multicolumn{2}{|c|}{ Vecindario } & \multicolumn{2}{|c|}{ Total } \\
\hline & & Media & Desv. Tip & Media & Desv. Tip & Media & Desv. Tip & Media & Desv. Tip & Media & Desv. Tip \\
\hline Casa-espacio abierto & 72 & 3,867 & 3,420 & 0,800 & 1,320 & 1,267 & 1,486 & 1,467 & 2,134 & 7,400 & 5,356 \\
\hline Casas & 15 & 3,850 & 2,877 & 0,940 & 1,177 & 1,329 & 1,738 & 0,550 & 0,945 & 6,669 & 4,211 \\
\hline Departamentos & 513 & 3,462 & 2,901 & 2,128 & 3,365 & 1,872 & 2,067 & 0,590 & 0,910 & 8,051 & 4,952 \\
\hline Instituciones-Industria & 39 & 2,958 & 2,382 & 0,792 & 1,233 & 1,611 & 1,835 & 0,861 & 1,335 & 6,222 & 4,084 \\
\hline Mercado-Comercio-Ofic & 101 & 2,129 & 2,171 & 1,347 & 1,652 & 1,733 & 1,838 & 0,545 & 1,044 & 5,753 & 4,158 \\
\hline Plaza & 122 & 2,992 & 2,541 & 1,475 & 1,824 & 1,402 & 1,761 & 0,484 & 1,070 & 6,353 & 4,197 \\
\hline Total & & 3,253 & & 1,080 & & 1,340 & & $\mathbf{0 , 5 3 0}$ & & 6,204 & \\
\hline
\end{tabular}

CUADRO 2

DESCRIPCIÓN ESTADÍSTICA DEL NÚMERO DE PERROS EN LA CALLE POR MANZANA, POR TIPO DE PERRO, SEGÚN FACTOR EN EL LUGAR, CIUDAD DE SANTIAGO, 2004

\begin{tabular}{|c|c|c|c|c|c|c|c|c|c|c|c|c|}
\hline \multirow[b]{2}{*}{ Factor } & \multirow[b]{2}{*}{ Existencia } & \multirow{2}{*}{$\begin{array}{c}N \\
864\end{array}$} & \multicolumn{2}{|c|}{ Callejero } & \multicolumn{2}{|c|}{ Supervisado } & \multicolumn{2}{|c|}{ Vagabundo } & \multicolumn{2}{|c|}{ Vecindario } & \multicolumn{2}{|c|}{ Total } \\
\hline & & & Media & Desv. Típ & Media & Desv. Típ & Media & Desv. Típ & Media & Desv. Típ & Media & Desv. Típ \\
\hline \multirow[t]{2}{*}{ Albergue } & $\mathrm{SI}$ & 382 & 3,712 & 2,900 & 1,207 & 1,531 & 1,895 & 2,029 & 0,762 & 1,205 & 7,576 & 4,445 \\
\hline & No & 482 & 3,222 & 2,671 & 1,017 & 1,535 & 1,066 & 1,446 & 0,446 & 0,881 & 5,751 & 3,924 \\
\hline \multirow[t]{2}{*}{ Agua } & si & 373 & 3,869 & 3,201 & 1,357 & 1,840 & 1,646 & 2,071 & 0,885 & 1,276 & 7,756 & 4,794 \\
\hline & No & 491 & 3,112 & 2,371 & 0,906 & 1,222 & 1,271 & 1,495 & 0,359 & 0,760 & 5,648 & 3,544 \\
\hline \multirow[t]{3}{*}{ Alimento } & si & 447 & 4,150 & 2,822 & 1,195 & 1,515 & 1,866 & 2,009 & 0,946 & 1,245 & 8,157 & 4,176 \\
\hline & No & 417 & 2,671 & 2,533 & 0,993 & 1,547 & 0,964 & 1,338 & 0,200 & 0,573 & 4,827 & 3,624 \\
\hline & Total & & 3,253 & & $\mathbf{1 , 0 8 0}$ & & 1,340 & & $\mathbf{0 , 5 3 0}$ & & 6,204 & \\
\hline
\end{tabular}

predominio sólo de casas, de casas-espacio abierto e instituciones-industrias.

Finalmente, desde el punto de vista del hábitat predominante en el lugar, en los perros de vecindario se distinguen dos grupos estadísticamente significati$\operatorname{vos}(\mathrm{p} \leq 0,05)$; el que incluye a casa-espacio abierto, instituciones-industria y departamentos con los promedios más altos (sobre 0,59 perros por manzana); $\mathrm{y}$ el grupo que incluye a casas, mercado-comercio-oficina y plazas con promedios bajo 0,55 perros promedio observados por manzana.

Se observaron diferencias significativas en el promedio de animales por manzana en lugares con existencia de albergue respecto de donde no existían, en el caso de perros callejeros, vagabundos y de vecindario (Cuadro 2), en que el promedio es mayor estadísticamente en lugares donde se observa un lugar de albergue. No se detectan diferencias, como es esperable, en el caso de perros supervisados en la calle.

$\mathrm{Al}$ analizar el tipo de albergue observado en el lugar (Cuadro 3), se observa en los perros callejeros promedios más altos asociados a la presencia de canchas y estructuras de la vía pública (como paraderos o jardines). En el caso de perros supervisados, como puede esperarse, los promedios no varían pues su presencia en el lugar no estaría asociada a la existencia de albergue. En el caso de perros vagabundos y de vecindario la existencia en el lugar de caniles y de estructuras en la vía pública marcan la diferencia.

$\mathrm{Al}$ considerar la disponibilidad o no de agua para beber en el lugar (Cuadro 2), se detectan diferencias significativas $(\mathrm{p} \leq 0,05)$ en el promedio de animales observados por manzana en los cuatro tipos de perro, siendo estadísticamente mayor en lugares donde se observa fuentes de agua para el animal.

El análisis por grupo de perro (Cuadro 4), considerando el tipo de fuente de agua para el perro identificado en las manzanas observadas, muestra diferencias en el promedio de animales por manzana en los cuatro grupos de perros. En el caso de perros callejeros su presencia es mayor si existen grifos o que las personas les provean agua. En los perros vagabundos la presencia de fuentes de agua y las personas, son las que marcan la diferencia. En los perros de vecindario la presencia de recipientes y las personas son las fuentes principales para este tipo de animal.

La existencia o no de fuentes de alimento para el perro, es uno de los factores que se considera más 
CUADRO 3

DESCRIPCIÓN ESTADÍSTICA DEL NÚMERO DE PERROS EN LA CALLE POR MANZANA, POR TIPO DE PERRO, SEGÚN LUGAR DE ALBERGUE, CIUDAD DE SANTIAGO, 2004

\begin{tabular}{|c|c|c|c|c|c|c|c|c|c|c|c|}
\hline \multirow{2}{*}{$\begin{array}{c}\text { Lugar } \\
\text { de albergue }\end{array}$} & \multirow{2}{*}{$\begin{array}{c}N \\
377\end{array}$} & \multicolumn{2}{|c|}{ Callejero } & \multicolumn{2}{|c|}{ Supervisado } & \multicolumn{2}{|c|}{ Vagabundo } & \multicolumn{2}{|c|}{ Vecindario } & \multicolumn{2}{|c|}{ Total } \\
\hline & & Media & Desv. Tip & Media & Desv. Tip & Media & Desv. Tip & Media & Desv. Tip & Media & Desv. Tip \\
\hline Canchas & 11 & 6,182 & 2,562 & 1,182 & 1,168 & 1,727 & 1,489 & 0,546 & 0,820 & 9,636 & 3,501 \\
\hline Canil & 27 & 5,185 & 2,949 & 1,000 & 1,074 & 2,482 & 2,901 & 1,667 & 1,240 & 10,333 & 4,243 \\
\hline Edificio & 41 & 3,561 & 2,550 & 1,439 & 1,534 & 2,073 & 1,836 & 0,512 & 0,840 & 7,585 & 3,931 \\
\hline Sitio Eriazo & 148 & 3,439 & 2,513 & 0,932 & 1,281 & 1,885 & 1,775 & 0,750 & 1,136 & 7,007 & 4,135 \\
\hline Jardines & 28 & 2,786 & 2,217 & 1,250 & 1,669 & 1,643 & 1,909 & 0,500 & 0,882 & 6,179 & 3,742 \\
\hline Plaza & 80 & 3,050 & 2,783 & 1,563 & 1,645 & 1,650 & 1,949 & 0,625 & 1,381 & 6,888 & 4,528 \\
\hline Vía pública & 42 & 5,286 & 4,002 & 1,476 & 2,155 & 2,119 & 2,698 & 0,905 & 1,186 & 9,786 & 5,340 \\
\hline Total & & $\mathbf{3 , 2 5 3}$ & & 1,080 & & 1,340 & & $\mathbf{0 , 5 3 0}$ & & 6,204 & \\
\hline
\end{tabular}

CUADRO 4

DESCRIPCIÓN ESTADÍSTICA DEL NÚMERO DE PERROS EN LA CALLE POR MANZANA, POR TIPO DE PERRO, SEGÚN FUENTE DE AGUA, CIUDAD DE SANTIAGO, 2004.

\begin{tabular}{|c|c|c|c|c|c|c|c|c|c|c|c|}
\hline \multirow{2}{*}{$\begin{array}{c}\text { Fuente de } \\
\text { agua }\end{array}$} & \multirow{2}{*}{$\begin{array}{c}N \\
372\end{array}$} & \multicolumn{2}{|c|}{ Callejeros } & \multicolumn{2}{|c|}{ Supervisado } & \multicolumn{2}{|c|}{ Vagabundo } & \multicolumn{2}{|c|}{ Vecindario } & \multicolumn{2}{|c|}{ Total } \\
\hline & & Media & Desv. Tip & Media & Desv. Tip & Media & Desv. Tip & Media & Desv. Tip & Media & Desv. Típ \\
\hline Acequia & 16 & 4,125 & 3,557 & 1,125 & 1,204 & 1,250 & 1,732 & 0,563 & 1,094 & 7,063 & 4,597 \\
\hline Fuente & 49 & 3,653 & 3,276 & 1,286 & 1,369 & 1,918 & 2,168 & 1,000 & 1,414 & 7,857 & 5,184 \\
\hline Grifo & 72 & 4,431 & 2,721 & 0,958 & 1,250 & 1,028 & 1,278 & 0,667 & 1,245 & 7,083 & 3,583 \\
\hline Personas & 67 & 4,955 & 3,431 & 1,478 & 1,531 & 3,090 & 2,838 & 1,821 & 1,266 & 11,343 & 4,538 \\
\hline Pozas & 116 & 3,017 & 3,349 & 1,767 & 2,596 & 1,129 & 1,585 & 0,233 & 0,565 & 6,147 & 4,814 \\
\hline Recipiente & 52 & 3,789 & 2,460 & 0,981 & 1,146 & 1,673 & 1,897 & 1,442 & 1,461 & 7,885 & 3,884 \\
\hline Total & & $\mathbf{3 , 2 5 3}$ & & 1,080 & & 1,340 & & $\mathbf{0 , 5 3 0}$ & & 6,204 & \\
\hline
\end{tabular}

\section{CUADRO 5}

DESCRIPCIÓN DEL NÚMERO DE PERROS EN LA CALLE POR MANZANA, POR TIPO DE PERRO, SEGÚN FUENTE DE ALIMENTO, CIUDAD DE SANTIAGO, 2004

\begin{tabular}{|c|c|c|c|c|c|c|c|c|c|c|c|}
\hline \multirow{2}{*}{$\begin{array}{l}\text { Fuente de } \\
\text { Alimento }\end{array}$} & \multirow{2}{*}{$\begin{array}{c}N \\
446\end{array}$} & \multicolumn{2}{|c|}{ Callejero } & \multicolumn{2}{|c|}{ Supervisado } & \multicolumn{2}{|c|}{ Vagabundo } & \multicolumn{2}{|c|}{ Vecindario } & \multicolumn{2}{|c|}{ Total } \\
\hline & & Media & Desv. Típ & Media & Desv. Típ & Media & Desv. Típ & Media & Desv. Típ & Media & Desv. Típ \\
\hline Basural & 48 & 4,583 & 2,857 & 0,917 & 1,366 & 2,500 & 1,857 & 0,479 & 0,922 & 8,479 & 4,136 \\
\hline Bolsas & 51 & 4,628 & 3,292 & 1,353 & 1,635 & 2,314 & 2,337 & 1,118 & 1,633 & 9,412 & 3,905 \\
\hline Locales & 65 & 3,062 & 2,888 & 1,877 & 2,388 & 1,339 & 1,417 & 0,615 & 0,930 & 6,892 & 4,473 \\
\hline Personas & 167 & 4,467 & 2,661 & 1,180 & 1,253 & 1,796 & 2,158 & 1,174 & 1,280 & 8,617 & 4,003 \\
\hline Recipiente & 115 & 3,930 & 2,635 & 0,887 & 1,066 & 1,817 & 1,899 & 0,922 & 1,193 & 7,557 & 4,166 \\
\hline Total & & $\mathbf{3 , 2 5 3}$ & & $\mathbf{1 , 0 8 0}$ & & $1, \mathbf{3 4 0}$ & & $\mathbf{0 , 5 3 0}$ & & 6,204 & \\
\hline
\end{tabular}

CUADRO 6

DESCRIPCIÓN ESTADÍSTICA DEL NÚMERO DE PERROS EN LA CALLE POR MANZANA, POR TIPO DE PERRO, SEGÚN SISTEMA DE DISPOSICIÓN DE LA BASURA, CIUDAD DE SANTIAGO, 2004

\begin{tabular}{|c|c|c|c|c|c|c|c|c|c|c|c|}
\hline \multirow{2}{*}{$\begin{array}{c}\text { Disposición de } \\
\text { la basura }\end{array}$} & \multirow{2}{*}{$\begin{array}{c}N \\
852\end{array}$} & \multicolumn{2}{|c|}{ Callejero } & \multicolumn{2}{|c|}{ Supervisado } & \multicolumn{2}{|c|}{ Vagabundo } & \multicolumn{2}{|c|}{ Vecindario } & \multicolumn{2}{|c|}{ Total } \\
\hline & & Media & Desv. Tip & Media & Desv. Tip & Media & Desv. Tip & Media & Desv. Tip & Media & Desv. Tip \\
\hline Acopio & 10 & 1,400 & 4,088 & 3,000 & 3,621 & 0,700 & 0,949 & 0,000 & 0,000 & 5,100 & 7,156 \\
\hline Bolsas & 543 & 3,917 & 2,737 & 1,035 & 1,328 & 1,473 & 1,690 & 0,674 & 1,059 & 7,099 & 4,062 \\
\hline Canasta & 81 & 2,432 & 2,525 & 0,963 & 1,156 & 1,037 & 1,735 & 0,259 & 0,685 & 4,691 & 3,777 \\
\hline Contenedor & 218 & 2,812 & 2,686 & 1,206 & 1,891 & 1,528 & 2,023 & 0,541 & 1,140 & 6,087 & 4,502 \\
\hline Total & & $\mathbf{3 , 2 5 3}$ & & 1,080 & & 1,340 & & $\mathbf{0 , 5 3 0}$ & & 6,204 & \\
\hline
\end{tabular}


importante para la presencia de estos animales en la calle. Se detectan diferencias estadísticamente significativas $(p \leq 0,05)$ en el promedio de animales por manzana (Cuadro 2), al comparar lugares con disponibilidad de alimento con aquellos clasificados sin fuente de alimento en el lugar, en el caso de perros callejeros, vagabundos y de vecindario. No se observan diferencias, como era de esperar, en el caso de perros supervisados, pues su presencia en la calle no es influida por la existencia o no de fuentes de alimento. Al considerar el promedio de perros totales, este es marcadamente mayor en lugares donde se observan fuentes de alimento $(8,1566)$, que donde no las hay $(4,8269)$.

Al clasificar las manzanas en que se observa disponibilidad de alimento en el lugar, de acuerdo a la fuente probable de alimento, se producen diferencias en los cuatro tipos de perro observados (Cuadro 5). En el caso de perros callejeros y vagabundos, su presencia es mayor en el caso que la basura se disponga en bolsas o existan micro basurales en el lugar. En los perros de vecindario el promedio es mayor en lugares donde se dispone la basura en bolsas y cuando las personas proporcionan alimento al animal.

La forma de la disposición de la basura para su posterior retiro por el servicio de recolección, es una variable que siempre se postula vinculada a la presencia de perros en las calles. La presencia de perros totales en la calle (Cuadro 6), es marcadamente mayor en aquellos lugares en que se deja la basura en bolsas $(7,0994)$ o en contenedores $(6,0872)$ y menor en lugares donde existen lugares de acopio cerrados $(5,1)$ o existen canastas en altura $(4,691)$. Específicamente en el caso de perros vagabundos, su presencia se asocia principalmente a la disposición de la basura en bolsas y contenedores y es menor en el caso que existan dispositivos de acopio o canastas dispuestas en altura. Llama la atención el alto número de perros asociado a la existencia de contenedores en el lugar, lo que se explica porque frecuentemente estos dispositivos son sobrepasados en su capacidad, observándose basura desparramada en su alrededor lo que pasa a constituir un polo de atracción para los perros por la posibilidad de encontrar restos de alimentos. En el caso de los perros supervisados, aparentemente podrían vincularse a lugares con dispositivos de acopio, lo cual podría explicarse en el hecho que el mayor número de perros supervisados se observó en comunas en donde existía una proporción importante de manzanas con predominio de departamentos, los cuales, disponen de lugares especiales de acopio de la basura. Esto mismo determinaría el bajo promedio en los otros tipos de perro observado en estos lugares.

Los resultados permiten concluir que la cantidad y tipo de perros observados en las calles de la ciudad de Santiago, se asocia a la existencia y tipo de lugares de albergue, fuentes de alimento y de agua existentes en la estructura urbana de la ciudad de Santiago. Así mismo, la presencia de perros se muestra asociada a la forma de disposición de la basura en las calles, siendo significativamente menor si hay dispositivos de acopio o canastas en altura que impidan el acceso de los animales a la basura.

\section{RESUMEN}

Con el objetivo de conocer que factores se relacionan con la presencia de perros en las calles, como contribución para la formulación de medidas sanitarias de vigilancia y control de la población canina, se realizó un estudio en 34 comunas de la Región Metropolitana, Chile, que conforman el gran Santiago. La unidad de muestreo fue la manzana y la información específica se obtuvo mediante la observación directa de 864 manzanas de la ciudad, asignando un mínimo de 25 manzanas por comuna elegidas al azar. Se registraron características de la población de perros observados en la calle y de la estructura urbana y del hábitat del lugar, para el estudio de la relación entre ellas.

Los resultados permiten concluir que la cantidad y tipo de perros observados en las calles de la ciudad de Santiago, se asocia a la existencia y al tipo de lugares de albergue, fuentes de alimento y de agua existentes en la estructura urbana de la ciudad de Santiago a las cuales el animal pueda tener acceso. Así mismo, la presencia de perros se muestra asociada a la forma de disposición de la basura en las calles, siendo significativamente menor si hay dispositivos de acopio o canastas en altura que impidan el acceso de los animales a la basura.

\section{REFERENCIAS}

Álvarez, E.; DOMíNGUEZ, J. (2001). Programa para el control integral de la población canina. Revista AMMVEPE, Ciudad de México. 12(3): 83-91.

Betzhold, A. (1998). Fecundidad Canina y Felina En el Gran Santiago, 1997. Tesis Med. Vet., Fac. Cs. Vet. y Pec., U. de Chile, Santiago, Chile. 110pp. 
IBARRA, L., MORALES, MA., ACUÑA, P. 2003". Aspectos demográficos de la población de perros y gatos en la ciudad de Santiago, Chile. Av Cs Vet 18, 13-20.

IBARRA, L., MORALES, MA., CÁCERES, L. 2003". Mordeduras a personas por ataque de perros en la ciudad de Santiago, Chile. Av Cs Vet 18, 41-46.

Morales, MA., IBARRA, L; CÁCERES, L. 2002. Morbilidad en perros en el sector urbano de la Región Metropolitana, Chile. Av Cs Vet 17, 28-33.
ORTEGA-PACHECO, A. (2001). La sobrepoblación canina: un problema con repercusiones para la salud humana. Revista Biomédica, Ciudad de México, 12(4): 290-291.

TAUCHER, E. 1997. Bioestadística. Editorial Universitaria S.A. Santiago, Chile. 309pp.

World Health Organization - World Society for the Protection of Animals (WHO -WSPA). 1990. Guía para el manejo de la población canina. Ginebra, Suiza. 128 pp. 